\title{
De la Diversidad a la Construcción de las Necesidades Educativas en las Aulas de Primaria. Un Análisis Interseccional desde el Caso Español
}

\author{
From Diversity to the Construction of Educational Needs in \\ Primary Schools. An Intersectional Analysis from Spain
}

\author{
Alejandro Paniagua * \\ Universidad Autónoma de Barcelona
}

\begin{abstract}
A partir de sus experiencias enseñando a tres niños y niñas identificados como “alumnado de necesidades educativas especiales" procedentes de familias lingüística, cultural y socioeconómicamente diversas, este artículo describe las ambigüedades a las que hacen frente varias maestras y maestros en relación con las necesidades de estos alumnos y alumnas. A través de las entrevistas y conversaciones mantenidas con estos profesionales se analiza la forma en las explicaciones "culturales" y "cognitivas" se relacionan y retroalimentan en sus narrativas. De este modo, el artículo muestra como una aproximación interseccional permite describir el carácter constituyente de unas necesidades que se creen a priori como objetivas educación especial, diversidad cultural-, y como éstas se entremezclan para generar formas genuinas de entender y aproximar la diversidad en el aula. En conclusión, se propone entender la Educación Especial como un sistema que deriva significado de todos los ejes de diferencia, reafirmándolos, y que tiende a ocultar la forma en que las necesidades del alumnado son construidas al margen de la responsabilidad de la institución escolar.
\end{abstract}

Descriptores: Educación especial, Diversidad cultural, España, Necesidades educativas.

Using their teaching experiences with children identified as having special education needs of linguistically, culturally and socioeconomically diverse families, this paper describes the ambiguities teachers face when understanding the needs of these students. To this extent, the informal conversations and interviews with teachers carried out during the observation of three classrooms are used to analyze the way in which 'cultural' and 'biological' explanations are intertwined and underpin their narratives. The paper discusses how an intersectional analysis allows to describe the constituent character of needs that are seen as objective special education, diversity-, and how these variables are mixed and produce new forms to understand the diversity in their classrooms. In conclusion, it is argued that special education may be understood as a system that derives meaning from all axes of difference, which are in turn reaffirmed, and which hides the way in which students' needs are constructed away from school's responsibility.

Keywords: Special needs education, Cultural diversity, Spain, Educational needs.

Esta investigación se realizó bajo el programa FPU 2010-2014 financiado por el Ministerio de Educación.

*Contacto: alejandro.paniagua@oecd.org

ISSN: 07 18-7378

www.rinace.net/rlei/
Recibido: $\quad 10 / 11 / 2016$

$1^{\text {a }}$ Evaluación: 12/03/2017

Aceptado: 22/03/2017 


\section{Revisión de la literatura}

Pese a que las ideas de raza y discapacidad han sido descritas como "ilusiones biológicas" que surgen de contextos culturales concretos (Everelles y Minear, 2010; McDermott y Varenne, 1995), estas ideas siguen estando firmemente ancladas en nuestras taxonomías cotidianas, en una sociedad siempre dispuesta a discriminar la diferencia (Artiles y Trent, 1994). En este artículo nos centramos en dos de las fuentes más importantes que configuran y nutren el mito del alumnado normal (Baglieri, Bejoian, Broderick, Connor y Valle, 2010) dentro de las escuelas, esto es, los parámetros estándar de funcionamiento considerados como normales (Artiles y Trent, 1994), y el ideal del blanco europeo (Reid y Knight, 2006). A partir de la existencia de estos estándares, se ha sugerido que el sistema de EE permite legitimar la actividad de los profesionales, a través de un discurso psico-médico que localiza la causa de los problemas de aprendizaje dentro del alumnado (Mehan, 2014; Skrtic, 2005). Existiría un proceso, señalan Artíles y Trent (1994), a través del cual la diversidad sería vista primero como desviación -a partir de su comparación con esos estándares- para luego, cargada con marcadores jerárquicos sobre lo que es más deseable y eficiente, ser finalmente asociada con ser menos capaz. Esto es lo que estos autores definen como "falsa analogía" y que explicaría por qué de manera frecuente, en el estado español, la dislexia se acaba convirtiendo en una discapacidad en vez de ser considerada una diferencia o, como mucho, una dificultad de aprendizaje (Blanco, 2009).

En la presente discusión se argumenta que la intersección de la diversidad cultural con el sistema de educación especial (EE) muestra la construcción subjetiva de las necesidades del alumnado. De manera general, las investigaciones en el estado español no han prestado demasiada atención a las situaciones en que las dificultades experimentadas por los alumnos familias LCSD $^{1}$ tienen que ver con n.e.e. ${ }^{2}$ no derivadas directamente de los procesos de incorporación y readaptación sociocultural (Carrasco, 2008). En algunos casos, la distancia socio-cultural y las dificultades de comunicación entre familias y profesionales puede retrasar diagnósticos que de lo contrario serían más rápidos para el alumnado autóctono. En otros, los profesionales pueden verse faltos de formación específica para identificar y distinguir los procesos adaptativos del alumnado de familias inmigradas de aquellos que corresponden a casos de n.e.e. (Paniagua, 2015). En relación con esta problemática intersección, Sales y colaboradores (2008) toman de un profesor británico una reflexión que podría servir como el punto de partida de este artículo:

Existe un problema con los niños que son de incorporación reciente y además necesitan EE: es difícil saber a qué grupos pertenecen (a los de refuerzo de inglés o a los de EE Tengo una pareja de niños polacos que seguramente sean de EE pero es muy difícil evaluarlos porque todavía no han aprendido la lengua (inglesa) tan rápido como los demás. (p. 30)

\footnotetext{
1 Se usa este término y no el genérico "inmigradas" para evitar generalizaciones imprecisas y para recalcar el impacto de las diferencias lingüísticas y el origen socioeconómico de éstas familias en las relaciones con los profesionales y la manera en que la escuela se aproximaba a las necesidades de sus hijos e hijas.

2 A lo largo del artículo se utiliza la idea de n.e.e. mayoritariamente como un marcador contextual que surge de los parámetros estandarizados y las expectativas de la escuela, pero también como descriptores objetivos, ya que muchos niños y niñas con dificultades - por no mencionar los casos claros de alumnado con discapacidades- requieren una atención diferenciada de los profesionales.
} 
Actualmente, el debate sobre los sesgos culturales de los test psicológicos todavía perdura (p. ej., Reynolds y Suzuki, 2012; Schellenberg, 2004), lo que muestra la complejidad que envuelve la manera en que las variables etno-culturales interaccionan con las dificultades de aprendizaje individuales (Artiles y Trent, 1994). Así, un estudio reciente alertaba sobre el peligroso efecto de las teorías monolingües persistentes en la escuela en relación al proceso de adquisición de la lectoescritura, bajo las cuales se tiende a ver a los lectores bilingües emergentes como alumnado con dificultades (Hopewell y Escamilla, 2014). Por otro lado, existe una creciente literatura que ha descrito las conceptualizaciones erróneas sobre la discapacidad y el peso de las teorías del déficit en el omnipresente discurso psico-médico de la mayoría de los profesionales (p. ej., Ahram, Fergus y Noguera, 2011; Harry y Klingner, 2006; Martínez Abellán, de Haro y Escarbajal, 2010).

Un ejemplo de este proceso de construcción de necesidades surge del estudio comparativo entre Estados Unidos y el estado español que realizan Harry y colaboradores (2008), donde ya nos avisan de las distintas connotaciones y consecuencias que se derivan de la noción de EE en ambos países: mientras que en Estados Unidos es más fácil que un alumno de bajo rendimiento sea ubicado en un programa de EE, en España estos programas estarían reservados al alumnado con discapacidad, ya que el alumnado de bajo rendimiento dispone de programas compensatorios propios. Sin embargo, más adelante alertan sobre como la amplia etiqueta "n.e.e.", que se aplica tanto al alumnado con discapacidad, al proveniente de familias LCSD, el de incorporación tardana o el que se identifica con dificultades de aprendizaje, podría predisponer al alumnado LCSD a usar, aunque fuera temporalmente, aquellos dispositivos pensados para mejorar la inclusión del alumnado con n.e.e.

Es necesario comprender estas ambigüedades en el contexto de la fallida implementación de la LOGSE, que se dio en paralelo a un fuerte crecimiento de la inmigración internacional en el estado español. La LOGSE, con una clara intención de desarrollar la "atención a la diversidad" en los centros y mejorar la inclusión de todo el alumnado, no consiguió cambiar la configuración del currículum, la pedagogía o el clima social de los centros educativos (Parrilla, 2007). Por el contrario, diversos estudios han puesto el acento en las contradicciones de las iniciativas para incluir al alumnado de n.e.e. o LCSD (Carrasco, Pàmies y Narciso, 2012; Martínez Abellán, de Haro y Escarbajal, 2010). Según estos autores, la creciente implementación de servicios externos y especializados habría provocado una progresiva implantación de una "lógica de externalización" (Poveda, Jociles y Franzé, 2014), que etiqueta y "disciplina” a los niños y niñas, condicionando su pertenencia a la escuela en función de su capacidad para adaptarse los requerimientos organizativos de la institución (Ríos-Rojas, 2014).

\section{Método}

Estudios recientes sobre el análisis interseccional han reclamado la necesidad de realizar una "aproximación constitutiva a las múltiples diferencias" (Yuval-Davis, 2006; citado en Everelles y Minear, 2010, p. 131) para comprender la combinación entre diversidad cultural y EE como algo más complejo que un análisis "sumativo" o "fragmentado" de los diferentes ejes de desigualdad. 
Como recogen Olmos y Rubio (2014), las relaciones entre múltiples variables pueden arrojar muchas variaciones en función de cómo se da cada intersección particular, sobre todo porque no siempre estas variables operan de igual manera. En el caso particular del alumnado LCSD identificado como n.e.e., los y las alumnas no son construidos primero como "culturalmente diversos" y luego definidos con arreglo a ideas sobre la discapacidad, ya que la misma idea de raza está cargada, social y culturalmente, con nociones de (in)competencia. De manera análoga, el sistema de EE surgió para filtrar las necesidades de todo el alumnado, necesidades que vienen dadas por como la idea de competencia o normalidad sanciona su diversidad acorde a esa "falsa analogía" descrita anteriormente. Si, como afirman Erevelles y Minear (2010), "la discapacidad es, de hecho, constitutiva del resto de diferencias sociales" (p. 133), la diversidad cultural no sería sino otra diferencia que permite "ver a los estudiantes extranjeros y aquéllos que viven en la pobreza como los otros gracias a su asociación con las n.e.e." (Reid y Knight, 2006, p. 18).

Partiendo de esta discusión, el artículo busca describir las relaciones entre estas dos variables para mostrar su carácter socialmente construido y poner a prueba esos supuestos imperativos psico-biológicos que otorgan un grado de neutralidad y objetividad a la construcción de las necesidades que se hace desde la institución escolar. Los datos presentados y analizados en las siguientes secciones provienen de la segunda fase de investigación de un proyecto que duro dos años y que tenía como objetivo reconstruir las experiencias de participación de familias LCSD con hijos e hijas identificados con n.e.e. Mientras que durante el primer año la investigación tuvo un carácter exploratorio y se entrevistaron a 23 familias y 19 profesionales, en el segundo año el trabajo de campo estuvo centrado en el análisis etnográfico de tres aulas de primaria, siguiendo los casos particulares de Masirent, Daniela e Iliase (Paniagua, 2015).

Para este artículo nos basamos principalmente en las entrevistas y conversaciones informales realizadas durante las observaciones de aula a las maestras y maestros que trabajaban con ellos, especialmente de Ángela -tutora de Masirent-, David -tutor de Daniela-, Bea y Mireia -tutoras de Iliase- y de sus respectivas maestras de EE así como otros profesionales del centro.

De manera específica, los casos aquí descritos de Masirent, Daniela e Iliase fueron seleccionados porque sus necesidades eran descritas de manera ambigua o eran casos poco claros de n.e.e., basados más en juicios profesionales que en datos biológicos verificables, y que permiten ver con mayor claridad "el rol de la cultura escolar en como la competencia y la incompetencia son definidos" (Harry y Klingner 2006, p. 8). Así, mientras a Masirent, llegado de Malí 3 años antes y alumno de tercero de primaria, se le había diagnosticado un retraso en la adquisición del lenguaje y un nivel por debajo de la media de su clase, Daniela, de madre dominicana que llevaba 6 años en la escuela, era descrita como muy poco atenta, con algún grado de dislexia, y con unas dificultades moderadas en todas las asignaturas de quinto curso. En su escuela, Siglo 21, estuve 5 meses y realicé 70 observaciones como asistente informal de David y Ángela, tutores de $5^{\mathrm{o}}$ y $2^{\mathrm{o}}$ de primaria (los nombres de las personas y las escuelas son pseudónimos). Siglo 21 era una escuela predominantemente catalana situada en un pueblo del área metropolitana de Barcelona. Tanto el pueblo como la escuela tenían menos de un 5\% de familias LCSD o recibiendo algún tipo de ayuda social. Pase una media de 1 o 2 días a la semana en ambas aulas en presencia de sus tutores o de otras maestras, en espacios comunes y reuniones, manteniendo conversaciones informales y abiertas con el 
profesorado. Dejando de lado las pocas horas que acudían a la clase de educación especial junto a otras personas, la mayoría de las intervenciones de la escuela consistían en respuestas individualizadas ad-hoc, ser menos exigentes con los trabajos o introducir pequeñas adaptaciones, especialmente en el caso de Daniela. A pesar de que el grupo de Daniela era considerado uno de los más complicados del centro, Ángela también manifestaba las dificultades que tenía para gobernar su clase. Precisamente era la tensa atmósfera en la clase de Daniela o la presencia de otros compañeros que eran vistos como más complicados lo que dificultaba que las maestras pudieran atenderles de manera más individualizada.

Finalmente, Iliase, nacido en Catalunya procedente de familia marroquí, tenía un problema de visión, muchas dificultades con la lectoescritura y un nivel muy por debajo de su clase de cuarto de primaria. Se realizaron visitas a la escuela El Valle durante 4 meses, observando un día a la semana la clase de Iliase, un niño de $4^{\circ}$ de padres marroquíes con un problema de visión y otras dificultades de aprendizaje, que iba muy retrasado en comparación con el nivel de la clase. La mayoría de las observaciones se hicieron con las dos tutoras al cargo de la clase -Bea y Mireia, quienes tenían una reducción de jornada- y con la maestra de educación especial, aunque también se pudo observar la clase con otros miembros del profesorado. Se realizaron un total de 35 observaciones en esta escuela situada en un área predominantemente trabajadora de una ciudad del área metropolitana de Barcelona. Debido a que la escuela estaba cerca de un barrio con una fuerte presencia de población inmigrada -representando alrededor de un 30\%-, el profesorado estaba acostumbrado a trabajar con alumnado LCSD, siendo éstos mayoría en la clase de Iliase. Comparado con los grupos de Masirent y Daniela, la clase de Iliase presentaba un clima mucho más complicado, donde los conflictos abiertos eran frecuentes, los cuales tenía un efecto devastador en la atención que Iliase recibía.

Las interacciones con los profesionales giraron en torno a una pregunta muy concreta: ¿cuáles son los retos y dificultades de enseñar al alumnado LCSD que está bajo el paraguas del sistema de EE? Gracias a mi experiencia previa como maestro, las relaciones con el profesorado fueron distendidas, siendo percibido mayoritariamente como un colega haciendo investigación para la universidad. Siguiendo a Casalta (2008), pese a que mi experiencia previa como maestro de primaria podía crear interferencias a la hora de observar y analizar la información, también es cierto que un uso reflexivo y crítico de mis disposiciones me permitieron revelar información muy sutil que podría haber pasado desapercibida para quienes no han tenido experiencia atendiendo a esta tipología de alumnado en la institución escolar. Por lo tanto, más que buscar explicaciones simples o culpabilizadoras, se procuró no someter las narrativas del profesorado a una crítica ideológica inmediata (Hammersley, 2006).

Todas las entrevistas fueron grabada y transcritas, mientras que las conversaciones informales que tuvieron lugar durante las observaciones fueron recogidas en el diario de campo. Ambas fuentes fueron codificadas de la misma forma, poniendo especial atención a las narrativas en torno a los argumentos "culturales" y los "biológicos", así como a otros temas fuertemente relacionados con ambos, como las familias y la diversidad cultural o la función de la EE y los soportes para la atención a la diversidad. Por otro lado, la interpretación de las narrativas de los profesionales se realizó a partir una aproximación basada en las "conversaciones internas" de las y los profesionales (Cornbleth, 2010), donde los individuos negocian sus opiniones y acciones entre sus ideales profesionales o personales y la naturaleza institucional de sus realidades 
cotidianas (Hammersley, 2006). De manera específica, se atendió a sus creencias y reflexiones en torno a la atención a la diversidad, la diversidad cultural y la existencia de dispositivos y/o ayudas para diferencias las necesidades derivadas de los procesos migratorios de aquéllas a las que tradicionalmente ha atendido el sistema de EE para fomentar un diálogo que hiciera hincapié en las superposiciones entre ambos tipos de necesidades y su relación con la institución escolar. Es en esa intersección donde el presente artículo hace hincapié, intentando mostrar hasta qué punto las conveniencias institucionales estaban relacionadas o daban forma a las intervenciones e interpretaciones de las y los profesionales observadas.

\section{Resultados}

\subsection{Contextos de aula}

Si no hubiera sido por las entrevistas y reuniones previas, Masirent habría sido descrito simplemente como un chico algo reservado que parecía tener pocas interacciones con sus compañeros, pero no un alumno de n.e.e. Durante el tiempo en que se le pudo observar en su escuela, nadie supo expresar de manera certera qué dificultades tenía. A pesar de reconocer su buen progreso desde que llegara a la escuela con 5 años, seguía recibiendo el apoyo de la maestra de EE porque aún tenía "algunas dificultades". El historial de Masirent ilustra de manera contundente el peso de las decisiones subjetivas. Así, la primera vez que se discutió su caso durante el año previo al trabajo de campo etnográfico, su maestra de EE le definió como un caso serio, incluyendo que iba muy retrasado en comparación con sus compañeros y compañeras y que le faltaban rutinas básicas y ciertas habilidades en relación con el proceso de lectoescriptura. En cambio, una vez se iniciaron las observaciones de aula, tanto su tutora -Ángela- como su nueva maestra de EE afirmaron que Masirent no debía considerarse un caso "real" de EE, sino un niño que recibía una ayuda extra que sería retirada tan pronto como no la necesitase. Más tarde alguien reconoció que el hecho de que comenzara a recibir EE fue porque "se asumió que quizás en casa no tenía la ayuda suficiente y que sus padres tenían una buena voluntad, pero no conocían el idioma de bien" (miembro del equipo directivo). Sobre este punto, Ángela manifestó que existía un tema más amplio relacionado con el contexto de su familia y que quizás sus padres no hacían todo lo posible para integrarse.

Masirent no había sido oficialmente escogido para realizar ninguna evaluación por parte del Equipo de Atención Psicopedagógica (EAP, encargados de evaluar y planificar las n.e.e.), ni tampoco seguía ningún Plan Individualizado (adaptación curricular), extremo que su tutora no acababa de descartar que sucediera más adelante, cuando las cosas refiriéndose al contenido curricular- se volviesen más "abstractas y complicadas". La clase de Masirent era una clase bien gestionada por parte de Ángela, que siempre conseguía motivar a su alumnado y favorecer un clima donde hubiera un diálogo distendido. Además, la tutora de Masirent tenía un interés explícito en favorecer una aproximación socio-emocional a sus alumnos. De esta forma demostraba tener, como mínimo, dos de los tres criterios que Harry y Klingner (2006) usan para definir la calidad de la pedagogía del profesorado, gestión de la clase y atender a la naturaleza socioemocional del aprendizaje, siendo el tercero la capacidad instructiva.

Por su parte, Daniela fue descrita desde el principio como una niña con "mucha imaginación”, ya fuera por su madre o por los profesionales con los que se mantuvieron 
las primeras reuniones. Como en los otros dos casos, las descripciones de Daniela evolucionaron durante el tiempo que duró la investigación, básicamente por lo que parecía ser una discrepancia entre sus pobres resultados académicos y un CI normal. Mientras sus profesores repetidamente insistían en su falta de atención y sus problemas de comportamiento y creían firmemente en que tenía algún tipo de dislexia, su madre solo reconocía que tenía algunas dificultades al identificar ciertas letras. Como alguna de sus maestras comentó, la demanda de intervención por parte del EAP se había ido retrasando durante años, y por consiguiente Daniela no había sido evaluada de manera correcta ni tampoco contaba con la ayuda especializada que su tutor creía que necesitaba. En este punto la disparidad de opiniones era clara: parte del profesorado pensaba que los servicios externos no habían priorizado su caso, en parte porque había otros casos más urgentes o importantes; mientras que otras personas no tenían reparos en señalar a su familia como poco proactiva. En una de las conversaciones que se mantuvo con David, su tutor, se reconoció que había existido un grave problema de comunicación con sus padres.

Para poner las cosas más difíciles, la clase de Daniela era descrita como una de las más difíciles de la escuela y cuando David no estaba en clase, ésta se convertía en una guerra sutil entre profesorado y estudiantes. En claro contraste, David mostraba una buena gestión de la clase, y su juventud y cercanía -así como ser su profesor de Educación Física- le permitían mostrarse simpático, pero a la vez riguroso y exigente con su grupo. Por otro lado, David intentaba siempre que podía introducir actividades colaborativas y que fueran interesantes y también ponía el acento en el clima de aula, un prerrequisito que él consideraba fundamental para favorecer que el alumnado pudiera trabajar siguiendo su propio ritmo. Sin embargo, Daniela solía sentarse cerca de alumnos que tenían mayores dificultades que ella, lo cual no hacía sino distraer aún más su atención. Como ocurriera con Masirent, en el caso de Daniela, aunque de manera más secundaria debido a su dislexia, la familia también aparecía como un argumento para explicar sus dificultades. De hecho, a pesar de que David comentó que durante los últimos seis meses solo se había entrevistado con su familia en una ocasión, en el informe de evaluación del 3er trimestre se recogía que "la familia no había asumido sus responsabilidades en casa", enfatizándose así la actitud negativa de sus padres, pero sin mencionarse los problemas que existían en el grupo de Daniela, la incapacidad del profesorado para atender a sus necesidades o ese problema de comunicación que se comentó anteriormente.

El caso de Iliase es de especial interés porque a pesar de los continuados esfuerzos de su maestra de EE, Raquel, y la buena colaboración de su familia, era el que presentaba los mayores problemas de los tres casos que se han descrito. En su clase se pudo observar el impacto negativo de una continua incapacidad por parte de sus tutoras de gestionar la clase, donde había broncas continuas y peleas entre el alumnado y entre algunos niños y niñas y los distintos profesionales. En un inicio, Iliase fue identificado con problemas de visión $y$, tras una intensiva intervención de un servicio privado externo, había continuado mostrando dificultades serias con el proceso de lectoescritura y una capacidad para distraerse muy elevada. Todo eso no había provocado la intervención del EAP durante cinco años, lo que a su vez había causado una cierta desafección por parte de Raquel y sus tutoras, Bea y Mireia.

A pesar de que Iliase fue descrito durante las primeras entrevistas como un alumno que no parecía tener ninguna dificultad a primera vista, durante la primera observación que pudo realizarse en su aula Iliase destacaba como uno de los niños más distraídos y 
perdidos de la clase. Más adelante, casi todo el mundo le describió como un estudiante "muy difícil" y que la escuela no sabía qué más hacer con él. Una de sus tutoras, Bea, hablaba abiertamente sobre su falta de tiempo para atender las necesidades educativas de Iliase, a pesar de que éste oficialmente estaba siguiendo un Plan Individualizado. De hecho, tanto Bea como Mireia gastaban casi todas sus energías en intentar retener el control de la clase, algo que pocas veces sucedía, de manera que su capacidad para centrarse en la pedagogía y la atención a la diversidad se veía reducida de manera drástica. Falto de alguien que le acompañara, Iliase constantemente perdía las tareas de casa y rara vez acababa el trabajo de clase. En contraste, pudo observarse el titánico esfuerzo de Raquel, que atendía a Iliase siempre con cariño en su aula de EE y a quien le intentaba adaptar todas las actividades. Por otro lado, Raquel había conseguido, usando su propio tiempo personal, asegurar los servicios externos de un centro de visión, en estrecha colaboración con su familia. Sorprendentemente, durante las evaluaciones trimestrales e incluso en la reunión de atención a la diversidad con la responsable del EAP del centro, su caso fue apenas comentado, ni tampoco el análisis de su progresión o su estancamiento- fue un tema discutido por los miembros de la comisión.

Aunque con diferencias notables entre los tres casos descritos, durante las observaciones de aula pudo observarse como en la mayoría de ocasiones Masirent, Daniela e Iliase estaban 'desconectados' de las dinámicas de la clase. En otro lugar se ha abordado en profundidad este tipo de situación como una forma de exclusión generada por la preeminencia de unas rutinas de aula que limitaban sus oportunidades para participar en la clase (Paniagua, en prensa). Aquí el énfasis se centra en cómo, a pesar de los esfuerzos de sus tutoras y tutores, los contextos de aula y la atención de los profesionales no respondía a las necesidades individuales de cada alumno.

Por un lado, los propios profesionales, especialmente en el caso de Masirent y Daniela, tenían la percepción que estaban haciendo bien su faena -aunque ello no estuviera exento de contradicciones-; mientras que, por otro, el hecho de que estos niños y niñas iban quedándose rezagados influía en la manera en que las maestras construían sus dificultades, las cuales tenían que gestionar dentro de los confines de la propia lógica de la institución escolar. Dichos confines están trazados por la existencia de unos servicios externalizados -fuera del aula ordinaria- diferenciados para alumnos de incorporación reciente $-\mathrm{O}$ inmigrantes- $\mathrm{y}$ alumnos de $\mathrm{EE}$, de manera que aquellos que se quedan a medio camino son los que se arriesgan a ser sobreatendidos, muchas veces de manera equivocada, como el alumnado bilingüe que es tratado con n.e.e., o a no ser atendidos, simplemente. Como se verá en el siguiente apartado, la dependencia hacia esos dispositivos especializados y las situaciones nuevas que genera esta intersección -n.e.e. de alumnos de familias LCSD no derivadas directamente de procesos de incorporación sociocultural- alimentaban esa "falsa analogía" entre diversidad y educación especial.

\subsection{La construcción de necesidades: ponderando las explicaciones "culturales" y “cognitivas"}

A pesar de la confianza generalizada hacia el modelo psico-médico mostrada por los profesionales (Paniagua, 2015) al intentar definir las necesidades del alumnado LCSD que mostraba dificultades de aprendizaje, Raquel afirmaba que "las cosas no eran tan simples". En estos casos, el alumnado corría el riesgo de quedar desatendido y ser identificado como de n.e.e. después ir quedándose rezagado durante años. De hecho, los tres casos discutidos en este artículo sufrieron retrasos en los procesos de evaluación 
externa y solamente Daniela había recibido un diagnóstico recientemente. Esos retrasos eran el resultado de una combinación de factores, que incluían "los diferentes prismas que utiliza cada maestra para observar las necesidades del alumnado", como manifestó Ángela; la falta de coordinación, especialmente durante las transiciones de curso; o la falta de involucración de las familias. Como se ha descrito en otro lugar (Paniagua, 2015), las relaciones de los profesionales con las familias LCSD eran complicadas. En concreto, las familias de estos tres casos afrontaban unas condiciones socioeconómicas precarias y tenían poca experiencia en la educación formal en sus respectivos países. Si a esto se le añade su a veces limitada capacidad para hablar en castellano o catalán (excepto en el caso de Daniela), el resultado es que para estas familias era complicado orientarse en el sistema educativo y comprender el detalle de las intervenciones de los profesionales.

Aunque a veces las descripciones que las tutoras y los tutores hacían de Masirent, Daniela e Iliase pudieran parecer negativas, nadie les consideraba como un alumnado inherentemente patológico. Es más, las maestras y los maestros asumían que sus dificultades podían atenderse con una correcta organización de los soportes, la aplicación de adaptaciones curriculares y una intervención coordinada. Por consiguiente, la mayoría aproximaba las etiquetas con precaución, como ponía de manifiesto Ángela:

Creo que a veces magnificamos las cosas... y es por eso que me gusta conocer la opinión de otros profesionales que trabajan con ellos.

Sin embargo, David, Ángela, y el resto de tutoras insistían constantemente en la falta de ayuda y orientación para acometer estas intervenciones. David, por ejemplo, mencionó un taller donde no recibió ninguna estrategia práctica y comentaba que los instructores "no conocen la realidad que tenemos en las aulas". Él creía que el reto principal era elaborar actividades donde todo el mundo pudiera participar a su manera, pero que esto resultaba "tremendamente complicado". Bea, siempre preocupada por su clase, manifestaba que en demasiadas ocasiones no recibía respuesta a sus preguntas y que estaba cansada de pedir soluciones a sus problemas. En un sistema que abiertamente confía en los discursos de los especialistas, las respuestas tendían a hacerse en base a localizar el problema en el mismo alumnado, ya que éste era el que al final tenía que adaptarse al contexto de aula y, especialmente, a la ausencia de soportes o adaptaciones. Sobre la falta de servicios especializados, Raquel mostraba dudas acerca de la conveniencia de los servicios existentes para atender a aquellos casos que no eran de incorporación reciente ni exactamente de EE.

Tendría que haber otro tipo de soporte para aquellos que llevan tres o cuatro años y continúan quedándose rezagados. Un soporte diferente al de EE que no puede ser ignorado, porque esas necesidades específicas ahora mismo están desatendidas.

Dado que estos profesionales no habían recibido una formación u orientación específica sobre la diversidad cultural, basaban sus respuestas a esta diversidad en su pertenencia como miembros de la cultura dominante, forjando una legitimación de la posición social del docente frente a la minoría (Carrasco, 2003). De este modo, los prejuicios y expectativas sesgadas formaban parte de sus visiones de las familias, tal y como se desprende del concepto de "familias cerradas" que usaban algunos profesionales para referirse a aquellas familias LCSD que eran aparentemente menos capaces de ayudar a sus hijos e hijas (Autor, 2015).

Mi sentimiento personal, que no he corroborado, es que la diferencia cultural es más importante que la clase social. Esto pasa con las familias árabes. No quieren 
malgastar su tiempo, traen a sus hijos a la escuela porque es obligatorio, pero... ¿Quién no ha traído los deberes hechos porque sus padres deberían haberla ayudado? La niña marroquí, otra vez.

Lejos de ser una visión testimonial o anecdótica, Carrasco, Pàmies y Bertran (2009) han descrito como la estructura de la escuela se presenta como muy inflexible y se adecúa poco a las realidades de las familias, a quienes su pertenencia étnica suele verse como un problema para la socialización de sus hijos e hijas. De hecho, la amalgama de factores familiares y contextuales suele vertebrarse, como veremos, en una narrativa que une lo cultural -que suele incorporar y ocultar las diferencias de clase-con las n.e.e.

El extremo de esa narrativa la podemos encontrar, por ejemplo, en el transcurso de una charla ofrecida por un psicólogo de un EAP sobre las necesidades del alumnado de familias LCSD. La cita es larga, pero merece la pena reproducirla en su totalidad:

$$
\begin{aligned}
& \text {...estos factores de riesgo [del contexto familiar] provocan un fenómeno que yo } \\
& \text { denomino, es un invento mío, provoca una 'deficientización', a ver si me explico: } \\
& \text { tenemos alumnos que no son deficientes ni mucho menos, pero el hecho de sufrir todas } \\
& \text { estas consecuencias... eh... le pasas un test y te sale deficiente, sale 'deficientado', no } \\
& \text { es deficiente, se ha 'deficientado' por falta de atención [en casa], falta de } \\
& \text { expectativas [de los padres], niños que se pasan muchas horas sin nadie, esto } \\
& \text { provoca una 'deficientización', es curioso. }
\end{aligned}
$$

Esta intervención recoge tímidamente -aunque de manera visiblemente racista- una idea elaborada hace tiempo por diversos autores que estudian la sobrerrepresentación en Estados Unidos (ej. Artiles y Trent, 1994; Blanchett, Klingner y Harry, 2009) y que es usada de manera mucho más cautelar y matizada por buena parte de los profesionales de las escuelas: que las condiciones socioculturales y económicas influyen en el desarrollo del alumnado de tal manera que posteriormente la escuela tiende a identificarlos como alumnado de EE, algo que los estudios en la Unión Europea comienzan también a verificar (ej. Berhanu y Dyson, 2012; Strand y Lindsay, 2009).

A pesar de estas ambigüedades y conexiones entre lo cultural y lo biológico, los maestros y maestras de Masirent, Daniela e Iliase parecían usar un sistema de oposiciones donde esas necesidades educativas especiales estaban o bien asociadas entorno a ideas de madurez o con cuestiones sociales y culturales. Estas taxonomías ayudaban precisamente a los profesionales a aproximar las ambigüedades provocadas por la intersección entre la EE y la diversidad cultural. Aplicado a los casos descritos aquí, estos dos sistemas de oposiciones vestían las narrativas en torno a las necesidades de Masirent, que se entendían como ocasionadas por problemáticas sociales y culturales, donde el contexto familiar, percibido desde la perspectiva del déficit, aparecía como variable principal. En el otro extremo aparecía Iliase, cuyas necesidades se ligaban a problemas de desarrollo, especialmente porque su familia mostraba una buena predisposición para colaborar y porque Iliase había nacido en Catalunya, cosa que hacía más improbables, aunque no inexistentes, las explicaciones de corte más cultural o social. El caso de Daniela caía en algún punto intermedio entre esos dos extremos. A pesar de estar diagnosticada de dislexia y que sus problemas de comportamiento eran más visibles, las visiones negativas acerca de su familia tenían una relevancia importante.

Una mirada más atenta, sin embargo, nos muestra como más allá de la superficie, las explicaciones culturales y las cognitivas funcionaban como un argumento auto sellado, con profundas conexiones: mientras los profesionales no veían a este alumnado como enteramente patológico -en parte porque eran conscientes de sus circunstancias 
socioeconómicas y culturales-, sí que daban forma a sus necesidades bajo el discurso de la Educación Especial, ya que no disponían de otras narrativas $-o$ servicios especializados-. Asimismo, en este proceso tendían a ver otras circunstancias asociadas a su contexto -como podría ser el bilingüismo, la adaptación sociocultural o su situación de clase precaria- como algo que tendía a ser problemático, lo cual a su vez reforzaba su visión de sus familias como disfuncionales o menos capaces. Por supuesto, el racismo biológico nunca es visible en los discursos populares o profesionales, pero el racismo cultural en la forma de visiones desde el déficit sigue impregnando la manera en que las escuelas “disciplinan” la diversidad cultural en el estado español (Ríos-Rojas 2014).

Volviendo a esa falsa analogía entre diversidad cultural y discapacidad, puede verse como esas dos categorías están conectadas, ya sea en la forma en que su pertenencia a familias LCSD 'pondera' el discurso psico-médico: "Masirent no es un caso serio de EE ... hay un problema cultural... su familia no le ayuda lo suficiente". Mientras que la hegemonía y dependencia del sistema de educación especial arrastraban la manera de entender sus necesidades educativas: "La familia de Daniela no la atiende como es debido... pero ella tiene un déficit de atención... dislexia”.

\section{Conclusiones}

Consistente con el análisis constitutivo de Everelles y Minear (2010), los tres casos presentados muestran como las intersecciones que emanan de la diversidad cultural y las n.e.e. se entremezclan y crean nuevas y complejas narrativas en torno a la diversidad y la desigualdad, que en el caso de Masirent, Daniela e Iliase tomaban la forma de una falta de atención de sus necesidades que ilustra los límites de su inclusión en el aula. Siguiendo el ya clásico trabajo de McDermott y Varenne (1995), esta intersección también describe la importancia de la organización de la escuela en la forma en que los problemas de aprendizaje son construidos y entendidos, pues la existencia de un soporte de EE y de las clases para el alumnado de incorporación reciente -especialmente designadas para atender los temas temporales, de adaptación y de lengua del alumnado LCSD- cosifican aún más este sistema de oposiciones. En particular, las lecturas culturales de los problemas de estos niños y niñas y la forma en que los profesionales usaban el etiquetaje objetivo y justo de la EE (Reid y Knight, 2006) permitían naturalizar y ocultar los prejuicios y sesgos en torno a las familias LCSD. Unas lecturas del contexto familiar que, al relacionarlas con sus n.e.e., permite describir la manera en que el sistema de EE deriva su significado de su intersección con cualquier eje de diferenciación, que en el caso que discutimos es la diversidad cultural.

Por lo tanto, podríamos sugerir que mientras el análisis interseccional muestra las asociaciones discursivas y sus consecuencias institucionales en la forma de falta de atención individualizada, es justamente el carácter indeseado de esta exclusión, por un lado, y la sutil y omnipresente presencia de los parámetros de normalidad que alimentan estas narrativas, por otro, lo que nos permitiría rehuir la aparente ausencia de ejes de desigualdad predominantes en el análisis interseccional. Al fin y al cabo, de lo que se trata es de dotar de significado a lo que no es normal, que aquí actúa como contingente y no como un contenido más. En este sentido, el análisis de las intersecciones que se dan en la educación especial permite ver con mayor claridad la equidistancia del discurso normativo ante cualquier eje de desigualdad, incluido la discapacidad -que aquí se entendería propiamente como contenido. No es, por tanto, simplemente atender a la 
relación compleja de los ejes de desigualdad, como se suele proponer en el análisis interseccional, sino entender cómo dichos ejes alimentan la constitución de la anormalidad o desviación -contingente-. ¿Sería el análisis interseccional solo un viaje para pasar de la antigua centralidad de la clase social a la centralidad de la (a)normalidad? De entrada, está posible conclusión permitiría quizás despoblar la a veces excesiva complejidad y arquitectura conceptual que ha ido acompañando al análisis interseccional para poder resituar el foco de análisis.

Desde el punto de vista de la educación inclusiva, esta reflexión tendría consecuencias importantes, en un contexto, como el español, donde no ha dejado de crecer el discurso especializado psicopedagógico, que sigue centrándose en identificar y responder de manera más discreta las necesidades específicas de los alumnos, pasando por alto el papel de la institución escolar en la construcción de las mismas, al creerlas siempre objetivas. Ante la falta de orientación y discursos opuestos a esta "falsa analogía" entre diversidad y discapacidad (Artiles y Trent, 1994), esto no hace sino menoscabar la confianza que tienen los profesionales de la capacidad del aula ordinaria para incluir toda la diversidad del alumnado mientras que, por el contrario, se alimenta esa "lógica de externalización" descrita por Poveda, Jociles y Franzé, (2014) que genera una visión benevolente de la EE.

En definitiva, este artículo apunta a la necesidad de desagregar los procesos adaptativos normales asociados a la inmigración de las dificultades específicas que caen dentro de la EE propiamente y pone el acento en el carácter institucional y subjetivo de la identificación y construcción de las necesidades del alumnado. Asimismo, alerta sobre cómo está intersección permite suavizar aparentemente la fuerza del discurso psicomédico a base de matizarlo con aspectos 'culturales' y de contexto, mientras dichos factores socioculturales son discriminados de manera 'objetiva' al apuntalarse por el rol de la EE, es decir, del discurso psico-médico. Ser conscientes de esto podría permitir repensar no solo la idoneidad y la visión benevolente de los servicios existentes, sino asumir la necesidad de volver a imaginar el aula ordinaria como el espacio por excelencia donde tienen que darse las respuestas a la diversidad, dentro de una lógica institucional escolar que asuma sus responsabilidades a la hora de construir y entender la diversidad.

\section{Referencias}

Ahram, R., Fergus, E. y Noguera, P. (2011). Addressing racial/ethnic disproportionality in special education: Case studies of suburban school districts. Teachers College Record, 113(10), 2233-2266.

Artiles, A. J. y Trent, S. C. (1994). The overrepresentation of minority students in special education: A continuing debate. The Journal of Special Education, 27(4), 410-437 doi: $10.1177 / 002246699402700404$

Baglieri, S., Bejoian, L. M., Broderick, A. A., Connor, D. J. y Valle, J. (2011). [Re]claiming 'inclusive education' toward cohesion in educational reform: Disability studies unravels the myth of the normal child. Teachers College Record, 113(10), 2122-2154.

Berhanu, G. y Dyson, S. (2012). Special education in Europe, overrepresentation of minority students. En J. Banks (Ed.), Encyclopedia of diversity (pp. 2070-2073). Thousand Oaks, CA: Sage. doi: 10.4135/9781452218533.n650 
Blanchet, W., Klinger, J. y Harry, B. (2009). The intersection of race, culture, language, and disability. Implications for urban research. Urban Education, 44(4), 389-409. doi: $10.1177 / 0042085909338686$

Blanco, A. I. (2009). Dislexia, escuela y exclusión social: un estudio desde la sociología acerca de la educación especial. Configurações, 5(6), 199-224.

Carrasco, S. (2003). Antropología de la educación y antropología para la educación. En A. González y J. L. Molina (Eds.), Abriendo surcos en la tierra. Investigación básica y aplicada en la $U A B$ (pp. 329-354). Bellaterra: UAB.

Carrasco, S. (2008). Segregació escolar i immigració: repensant debats i alternatives. Revista Nous Horitzons, 191, 31-40.

Carrasco, S., Pàmies, J. y Bertran, M. (2009). Familias inmigrantes y escuela: Desencuentros, estrategias y capital social. Revista Complutense de Educación, 20(1), 55-78.

Carrasco, S., Pàmies, J. y Narciso, L. (2012). A propósito de la acogida de alumnado extranjero. Paradojas de la Educación Inclusiva en Cataluña (España). Revista Latinoamericana de Educación Inclusiva, 6(1), 105-122.

Casalta, V. (2008). De les pràctiques escolars alas habitus docents: La importancia de les figures dels $i$ les docents en la integració dels fills i filles de famílies immigrants a les escoles. Licencia de estudios. Barcelona: Departament d'Educació.

Cornbleth, C. (2010). Institutional Habitus as the de facto diversity curriculum of teacher education. Anthropology and Education Quarterly, 41(3), 280-297. doi: 10.1111/j.15481492.2010.01088.x

Erevelles, N. y Minear, A. (2006). Unspeakable offenses: untangling race and disability in discourses of intersectionality. Journal of Literary $\Xi^{2}$ Cultural Disability Studies, 4(2), 12745. doi: $10.3828 /$ jlcds.2010.11

Hammersley, M. (2006). Ethnography: problems and prospects. Ethnography and Education, 1(1), 3-14. doi: 10.1080/17457820500512697

Harry, B., Arnaiz, P., Klingner, J. K. y Sturges, K. (2008). Schooling and the construction of identity among minority students in Spain and the U.S. Journal of Special Education, 42(1), 15-25. doi:10.1177/0022466907313605

Harry, B. y Klingner, J. (2006). Why are so many students in special education? Understanding race y disability in schools. Nueva York, NY: Teachers College Press.

Hopewell, S. y Escamilla, K. (2014). Struggling reader or emerging biliterate student? Reevaluating the criteria for labeling emerging bilingual students as less achieving. Journal of Literacy Research, 46(1), 68-89. doi: 10.1177/1086296X13504869

Martínez Abellán, R., de Haro, R. y Escarbajal, A. (2010). Una aproximación a la educación inclusiva en España. Revista de Educación Inclusiva, 3(1), 149-164.

Mehan, H. (2014). The prevalence and use of the psychological-medical discourse in special education. International Journal of Educational Research, 63, 59-62. doi: 10.1016/j.ijer.2012.10.003

McDermott, R. y Varenne, H. (1995). Culture as disability. Antrhopology and Education Quarterly, 26, 323-348. doi: 10.1525/aeq.1995.26.3.05x0936z

Olmos, A. y Rubio, M. (2014). Imaginarios sociales sobre 'la/el buen y la/el mal estudiante'. En P. Cucalón (Ed.), Etnografía Escolar e interseccionalidad (pp. 9-15). Madrid: Traficantes de sueños. 
Paniagua, A. (2015). The participation of immigrant families with children with SEN in schools: a qualitative study in the area of Barcelona. European Journal of Special Needs Education, $30(1), 47-60$.

Paniagua, A. (en prensa). The intersection of cultural diversity and special education in Catalonia: the subtle production of exclusion through classroom routines. Anthropology \& Education Quarterly.

Parrilla, A. (2007). Inclusive Education in Spain: a view from inside. En L. Barton y F. Armstrong (Eds.), Policy, Experience and Change: Cross-Cultural Reflections on Inclusive Education (pp. 19-36). Dordrecht: Springer.

Poveda, D., Jociles, M. I. y Franzé, A. (2014). Immigrant students and the ecology of externalization in a secondary school in Spain. Anthropology y Education Quarterly, 45(2), 185-202. doi:185-202. 10.1111/aeq.12058

Reid, D. K. y Knight, M. G. (2006). Disability justifies exclusion of minority students: A critical history grounded in disability studies. Educational Researcher, 35(6), 18-23. doi:10.3102/0013189X035006018

Reynolds, C. R. y Suzuki, L. A. (2012). Bias in psychological assessment: An empirical review and recommendations. En I. B. Weiner, J. R. Graham y J. A. Naglieri (Eds.), Handbook of psychology: Assessment psychology (pp. 82-113). Hoboken, NJ: Wiley. doi:10.1002/9781118133880.hop2 10004

Ríos-Rojas, A. (2014). Managing and disciplining diversity: The politics of conditional belonging in a Catalonian institute. Anthropology y Education Quarterly, 45(1), 2-21. doi: 10.1111 /aeq.12044

Sales, R., Ryan, L., Lopez, M. y D’Angelo, A. (2008). Polish pupils in London schools: Opportunities and challenges. Londres: Middlesex University.

Schellenberg, S. J. (abril, 2004). Test bias or cultural bias: Have we really learned anything? Comunicación presentada en el Meeting of the National Council for Measurement in Education. San Diego, California.

Skrtic, T. M. (2005). A political economy of learning disabilities. Learning Disability Quarterly, 28,149-155. doi:10.2307/1593616

Strand, S. y Lindsay, G. (2009). Ethnic disproportionality in special education: evidence from an english population study. Journal of Special Education 43(3), 174-190.

\section{Breve CV del autor}

\section{Alejandro Paniagua}

Doctor en Antropología Social por la Universidad Autónoma de Barcelona (UAB). Actualmente trabaja como investigador postdoctoral en el Center for Educational Research and Innovation (CERI) en la OCDE en París, mientras sigue vinculado al grupo de investigación EMIGRA-CER de la UAB. Ha hecho estancias de investigación en Londres y Los Ángeles. Su investigación gira en torno a la participación, la innovación, la diversidad y el análisis institucional de las prácticas docentes. Durante más de 7 años trabajó como maestro en centros de primaria situados en el área metropolitana de Barcelona. ORCID ID: 0000-0002-7794-8321. Email: alejandro.paniagua@oecd.org 